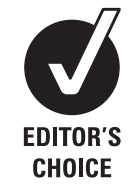

${ }^{1}$ Royal Hospital for Sick Children, Yorkhill, Glasgow, UK

${ }^{2}$ Vision Sciences, Glasgow Caledonian University, Glasgow, UK

${ }^{3}$ Glasgow Caledonian University, London, UK

${ }^{4}$ Royal Alexandra Hospital,

Paisley, UK

Correspondence to

Mr Richard Bowman,

Ophthalmologist, Royal Hospital for Sick Children, Dalnair Street, Yorkhill, Glasgow G3 8SJ, UK; richardandruthbowman@gmail. com

Accepted 19 October 2009

Published Online First

30 November 2009

\title{
The 'mirror test' for estimating visual acuity in infants
}

\author{
Richard Bowman, ${ }^{1}$ Daphne L McCulloch, ${ }^{2}$ Elizabeth Law, ${ }^{3}$ Karen Mostyn, ${ }^{4}$ \\ Gordon N Dutton ${ }^{2}$
}

\section{ABSTRACT}

Objective The authors aimed to investigate the association between the distance at which infants fixate their own reflections and visual acuity card testing, and to determine whether this could form the basis of a new clinical test of visual function in infants.

Methods 78 healthy infants under 9 months of age (range 1-266 days, mean 56.5 564 SD) were recruited and held close to a mirror such that they attended their own reflections. The distance from the mirror was increased until they no longer held fixation. Binocular acuities were tested with the Teller acuity card procedure.

Results Reliability was rated 'good' in 58 and 60 infants respectively, for mirror distances and for the acuity cards. Data were also included for moderate reliability ( $n=20$ and 14 respectively). The mean mirror distance was $54.9 \mathrm{~cm}$ (range 13.5-178, SD=42.8). The mean Teller acuity was 2.19 cycles per degree (range $0.2-14.5, S D=2.8$ ). (Snellen equivalent 6/82, range 6/ $900-6 / 12)$. Mirror distance showed linear correlation with both Teller acuity $\left(R^{2}=0.69, p<0.0005\right)$ and with age $\left(R^{2}=0.73, p<0.0005\right)$ by univariate analysis. Using multivariate analysis, only age retained significance. Using logarithmic scales and a logistic growth function for age, correlations were stronger (log mirror distance vs log Teller acuity, $R^{2}=0.86, p<0.0005$; logistic regression of log mirror distance vs log age, $R^{2}=0.88$, $p<0.0005)$, and both retained independent significance in a multivariate model.

Conclusion Mirror fixation distance increases with age in infants and has a good correlation with acuity card results. The portability and ease of use would make it a useful additional tool for detecting impaired visual function in infants.

\section{INTRODUCTION}

Assessing visual acuity in infants may be important in early detection of treatable conditions that may cause irreversible amblyopia if missed, such as cataract or high refractive error. Such an assessment is challenging, since infants cannot communicate what they are seeing. Face recognition is not routinely ascertained, even in those who can communicate, and its relationships with standard metrics of vision are not well understood.

Acuity card techniques, which are widely used, depend on infants' preference to look at patterns rather than homogeneous fields. ${ }^{1}$ These tests require specialist equipment and skills. Infants make differential responses to faces or face-like patterns compared with similarly detailed non-face stimuli. ${ }^{2}$ It seems logical to utilise this preference to facilitate rapid acuity assessment in this age group.
We have observed that infants will attend to the reflection of their own faces in a mirror and that attention is lost at progressively greater distances with increasing age. Using only a mirror and the infants' interest in their own reflections, we designed a simple test for infants and compared this with a standard acuity card test.

\section{PARTICIPANTS AND METHODS}

Infant participants were recruited from the postnatal ward and at postnatal physiotherapy classes at the Queen Mother's Hospital, Glasgow. An undilated retinoscopy, a Hirschberg test (looking for symmetrical corneal reflections of a pen torch) and a brief history were used to exclude children born more than 3 weeks prematurely or those with obvious ophthalmological or refractive abnormalities. For both acuity card and mirror tests, the infant was supported sitting on the mother's lap with the infant facing a wall with the minimum of distracting objects. A portable screen and/or side shields on the mirror were used in the physiotherapy room. Approval was given by the Ethical Committee of the Yorkhill Hospitals, NHS Trust, and the mothers gave informed consent for their infants to participate.

\section{Teller acuity card test}

Teller acuity cards have a grating of black and white stripes presented on one side of a grey card. If the grating is visible, the child will preferentially attend the patterned side, and the observer, masked to the location of the grating, will notice the direction of gaze (from behind the card through a peephole). The average brightness of the grating is matched to that of the background grey to avoid any confounding effect. The spatial frequency of squarewave gratings vary in from 0.32 to 38.0 cycles $/ \mathrm{cm}$ ( 1 cycle $=1$ black and white stripe) and occupy an area of $12.5 \times 12.5 \mathrm{~cm}$ to the side of a central peephole on the rectangular Teller cards $(25.5 \times 51 \mathrm{~cm})$

For this study, the method described by Teller ${ }^{4}$ was used. Infants less than 7 months of age were tested at $38 \mathrm{~cm}$, and the first card used was 0.64 cycles/degree. (This coarse grating has a $\log$ MAR equivalent resolution of 1.67 or $1-\log M A R$ of -0.67; Snellen equivalents of $6 / 280$ metric or $20 /$ 940 feet). Children between 7 and 18 months were tested at $55 \mathrm{~cm}$ and initially presented with 1.3 cycles/degree (logMAR 1.36, Snellen 6/140). Both initial targets should be easily detectable and allow the examiner to assess the child's general attentiveness. The card was spun face down to mask the observer, who made a judgement as to which way the child looked through the peephole then looked to see if it corresponded to the grating. The process (with spinning of the card) was 
repeated twice more, and two 'correct' responses were interpreted to mean that the grating was seen. Once the threshold was reached, and the response stopped, a coarser grating was tried again to ensure that the infant had not simply lost interest.

\section{Mirror test}

Infants were held approximately $20 \mathrm{~cm}$ from a mirror until they were deemed to attend their own reflection. The child was then moved slowly back until fixation was lost, as evidenced by the head and eyes turning away. The mirror-to-child distance was measured with a tape measure. (Note that because of reflection, the actual viewing distance is twice the distance from the child to the mirror.) The process was carried out five times; the low and high values were discarded, and the mean of the three other values was calculated as the threshold. Two different mirror arrangements were used: a hand-held mirror and a wallmounted mirror. For the wall-mounted mirror, the top part was shielded to block out the mother's face. A mirror size of approximately $30 \mathrm{~cm}$ by $40 \mathrm{~cm}$ was found to avoid any practical problems of loss of face reflection due to image decentration as viewing distance increased.

For both acuity cards and mirror testing, the child's behaviour and attention were recorded. If the infant was alert and attentive throughout, attention was recorded as good. The responses of those who fell asleep, became distressed or otherwise did not complete the test were regarded as poor and excluded from further analysis.

\section{RESULTS}

Eighty-one healthy infants (mean age 56.5 days (range $=1-266$, $\mathrm{SD}=64.2)$ ) were recruited. Attention was rated 'good' in 58 and
60 infants respectively, for mirror distances and for the acuity cards. Data were also included for moderate attentiveness $(n=20$ and 14, respectively). The hand-held mirror was used for 55 infants (68\%), the wall-mounted mirror for 26 (32\%). Good attention to the mirror test was recorded for $24 / 26$ (92\%) children using the wall-mounted mirror, a better proportion than with the hand-held mirror $(33 / 55,62 \%)\left(\chi^{2}=8.19, \mathrm{p}=0.017\right)$.

The mean mirror distance was $54.9 \mathrm{~cm}$ (range 13.5-178, $\mathrm{SD}=42.8)$. The mean acuity for gratings using the Teller test was 2.19 cycles per degree (range $0.2-14.5, \mathrm{SD}=2.8$ ). Mirror distance showed a linear correlation with both Teller acuity $\left(R^{2}=0.69\right.$, $p<0.0005)$ and age $\left(R^{2}=0.73, p<0.0005\right)$ by univariate analysis. Using the multivariate analysis, age retained significance. Using logarithmic scales, the correlation with Teller acuity was stronger (log mirror distance vs log Teller acuity, $\mathrm{R}^{2}=0.86$, $\mathrm{p}<0.0005$ (figure 1)) (log mirror distance vs log age $\mathrm{R}^{2}=0.85$, $\mathrm{p}<0.0005)$; both retained independent significance in a multivariate model. Conversions to equivalent Snellen, logMAR and decimal acuity are also given in figure 1. Analysis of the subgroup with the hand-held mirror revealed a similar correlation between log mirror distance and log Teller acuity $\left(R^{2}=0.86\right.$, $\mathrm{p}<0.0005)$, but there was no significant correlation if only the smaller group with the wall-mounted mirror test was considered.

The association between age and mirror distance was best fitted using regression of a curvalinear function (the logistic growth function: mirror distance $(\mathrm{cm})=($ adult distance $\times$ age $(\mathrm{da})) /$ $(\sigma+$ Age $)+b$, where distance and age are on a logarithmic scale, $b$ is the mirror distance for a neonate, and $\sigma$ is the age at which the distance is half of the adult distance) with the logarithm of mirror distance $\left(\mathrm{R}^{2}=0.89, \mathrm{p}<0.0001\right)$ as shown in figure 2 .
Figure 1 Mean mirror fixation distance for 81 healthy infants from birth to 9 months of age, and for 10 adults with corrected visual acuity (VA) of logMAR -0.16 to $+0.02(6 / 4.5$ to $6 / 6$ - metric Snellen). Adults moved towards a mirror in a corridor until their faces were recognisable. The curves show the least-squares fits for logistic growth curves for the mean (typical) and the lower $95 \% \mathrm{Cl}$ with representative values given in table 1 . cpd, cycles per degree.

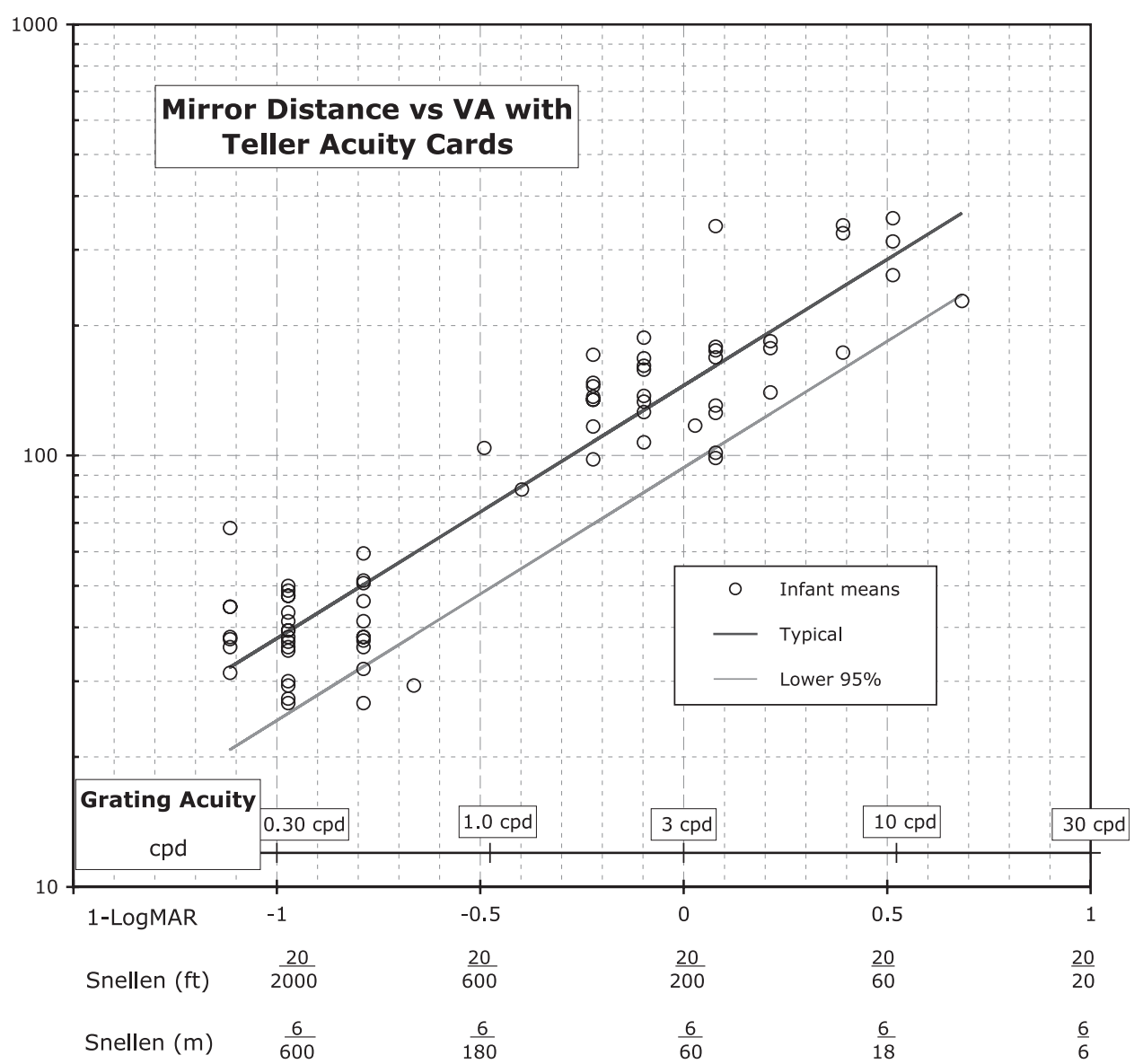


Figure 2 Visual thresholds with the Teller acuity card test compared with the mirror fixation distance for 74 infants who were attentive for both tests. The bold line is the best-fit linear regression using the logMAR acuity versus the logarithm of the mirror distance. The lower $95 \% \mathrm{Cl}$ is also shown. Expected values of Teller acuity for representative mirror distances given in table 1 are based on these regression lines. mo, months.

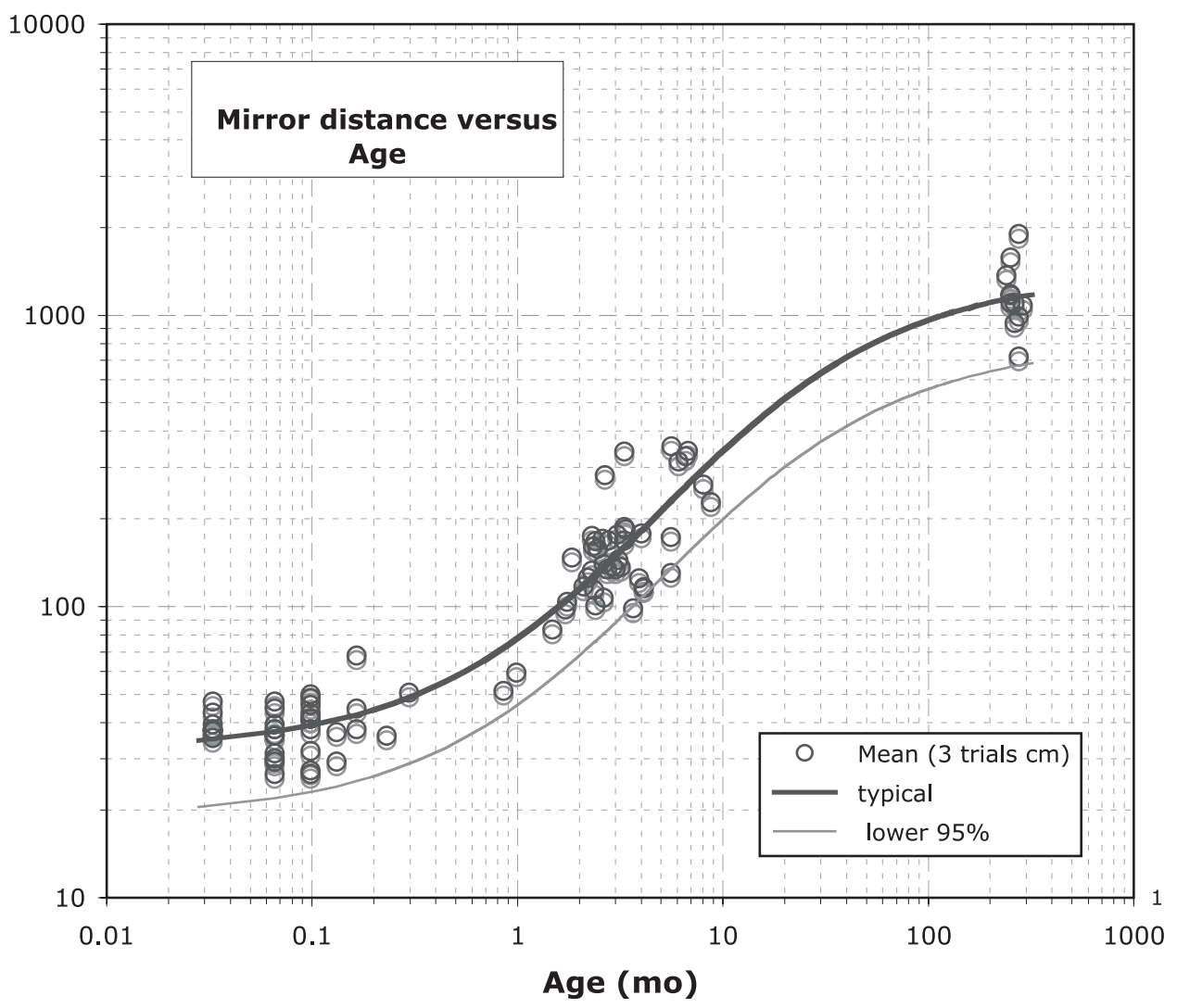

Typical (mean) mirror distances and associated Teller acuities at different ages and the lower limits expected are given in table 1. Both means and limits of agreement were calculated from the best-fitting regression lines illustrated in figures 1, 2 .

\section{DISCUSSION}

Measurement of the 'mirror distance' provides a novel technique for visual acuity estimation in infants. It is simple and reliable, and shows a good correlation with the standard acuity card technique.

Although attention to the wall-mounted mirror was better than for the hand-held mirror, small numbers and limited age range within this subgroup precluded useful subgroup analysis. Further work, to investigate and standardise the technique with wall-mounted mirrors, to compare responses with control nonreflective surfaces, to investigate monocular responses and to apply the test to children with eye conditions leading to reduced visual acuities, is indicated.

Atkinson conducted a meta-analysis of studies using the Teller acuity test in infants and found that, as an approximate rule of thumb, age in months equated to number of cycles/degree up to 12 months. ${ }^{5}$ However, she also stated that some studies indicated that the rate of increase in acuity slows down before 12 months. The latter is consistent with these data that follow the same 'rule of thumb' from 2 to 4 months (see table 1 ). These data are better fit when using a logarithmic scale for age, indicating non-linear development (ie, acuity development slows within the age range tested, yielding lower rates of improvement in older infants). Habituation (loss of interest in a stable image) is a possible source of underestimating visual acuity with our method of slowly increasing mirror distance. As habituation generally increases with age and with neural development, it could also account for underestimated acuities in older children.
Alternatively, development of the ability to perceive facial detail may mature more slowly than grating acuity in older infants.

It is not clear whether the high contrast acuity task required for acuity card tests relates directly to face perception. Some adult studies report that high-contrast visual tasks are the best predictors of face recognition, ${ }^{6}{ }^{7}$ while others find stronger associations with low-contrast tasks. ${ }^{8-10}$ In a recent populationbased study (the SEE study), both contrast sensitivity and visual acuity were significant independent predictors for a facerecognition task. ${ }^{11}$

Two phases in the development of contrast sensitivity have been reported. ${ }^{5}$ Between 4 and 9 weeks, the overall contrast

Table 1 Equivalent grating, Snellen and mirror distance acuities for different ages

\begin{tabular}{|c|c|c|c|c|}
\hline Age (days) & $\begin{array}{l}\text { Teller acuity } \\
\text { (cycles/ } \\
\text { degree) }\end{array}$ & $\begin{array}{l}\text { Snellen } \\
\text { acuity } \\
\text { equivalent }\end{array}$ & $\begin{array}{l}\text { Mirror distance } \\
\text { (cm) (fixation/ } \\
\text { reflected distance } \\
(2 \times))\end{array}$ & $\begin{array}{l}\text { 95th percentile } \\
\text { lower confidence } \\
\text { limit }(\mathrm{cm})\end{array}$ \\
\hline $1.29(31 \mathrm{~h})$ & 0.33 & $6 / 540$ & $15.5(31)$ & $13(26)$ \\
\hline $\begin{array}{l}14.1 \\
\text { (2 weeks) }\end{array}$ & 1 & $6 / 180$ & $38.2(76.4)$ & $24.5(49)$ \\
\hline $\begin{array}{l}43.7 \\
\text { ( } 6 \text { weeks) }\end{array}$ & 2 & $6 / 90$ & $57.2(115)$ & $37(74)$ \\
\hline $\begin{array}{l}79.4 \\
\text { (11 weeks) }\end{array}$ & 3 & $6 / 60$ & $72.5(145)$ & 47 (94) \\
\hline $\begin{array}{l}126 \\
\text { (4.2 months) }\end{array}$ & 4 & $6 / 45$ & 85.5 (171) & 55.5 (111) \\
\hline $\begin{array}{l}177 \\
\text { (5.8 months) }\end{array}$ & 5 & $6 / 36$ & 97.5 (195) & $62.5(125)$ \\
\hline $\begin{array}{l}{ }^{*} 512 \\
\text { (17 months) }\end{array}$ & 10 & $6 / 18$ & $147(294)$ & 93.5 (187) \\
\hline $\begin{array}{l}\text { *2951 } \\
\text { (8 years) }\end{array}$ & 30 & $6 / 6$ & $279(558)$ & $180.5(361)$ \\
\hline
\end{tabular}

\footnotetext{
*Extrapolated beyond the age range of the study subjects.
} 
sensitivity increased by a factor of 4-5 across all spatial frequencies. Beyond 9 weeks, the contrast sensitivity at low spatial frequencies remained constant, while sensitivity increased systematically at higher spatial frequencies.

Developmental psychology work shows that mirror self recognition is rarely reported before 2 years. ${ }^{12}$ These data, showing correlation of mirror distance with both age and grating acuity, suggest that the primary stilling response to the mirror self image is a resolution acuity response and therefore not confounded by the complexities of self recognition.

It is possible that the mirror test may offer no advantages over using another face (eg, mother's or clinician's) as the target. The mirror does, however, offer certain theoretical advantages: the mirror itself limits background distractions; infants tend to stare when they attend their own face, limiting variables such as motion and sound that would be tempting for an adult to insert when trying to gain fixation; the actual distances are doubled by the mirror making it convenient and again reducing the background size.

There is clearly a limit to the precision of the technique (our $95 \%$ CIs for mirror distance overlap between $6 / 90$ and $6 / 36$ and also between $6 / 18$ and $6 / 60$ ), but this is probably inevitable for infant psychophysical techniques and is also true of acuity card techniques where Teller reported variation by a factor of $2 .{ }^{4}$ Although the mirror test may seem more subjective than preferential looking techniques with targets of known size and contrast, the Teller acuity test is also subjective; the examiner uses all cues to judge whether the gratings are seen or unseen, and if so in which position. The 'objective' forced choice preferential looking strategy is time-consuming and requires several visits per infant to statistically 'prove' the viewing preference. ${ }^{13}$

We believe this technique may have a number of useful applications. It would be easily conducted at home and could be used as a screening technique by a variety of health professionals and even parents. Health professionals have traditionally used fixing and pursuit (following) eye movements ( $F \& F)$ to targets of known size and colour. Although these have been shown to correlate with a validated test such as Teller, they are subjective judgements that have been validated only for paediatric ophthalmologists. ${ }^{14} 15$ In adults, traditional informal measures of acuity in adults are associated with a wide range of resolution acuity levels. ${ }^{16}$

Though further work is needed to refine the mirror test, as outlined above, it may prove useful as an adjunct to an eye examination (more brief tests work better than relying on fewer tests with infants), as an adjunct to GP and health visitor's screenings that are already in place. These data suggest that this would improve the sensitivity and specificity of referrals compared with poor F\&F or visual attention. The mirror test also provides an easy way to demonstrate degree of visual impairment to parents and illustrate its effect in terms of seeing everyday visual targets at different distances. Therefore, while this test cannot replace the standard techniques, it may be useful in situations where standard equipment is not available, or when a quick estimation is required for screening purposes.

Acknowledgements We thank $\mathrm{R}$ Hamilton, for fitting the logistic growth curves and calculating the confidence limits, and NJ Hagan and MW Allen, for collecting the adult data illustrated.

Funding Eye Research for Children Fund (Greater Glasgow Health Board).

Competing interests None.

Patient consent Obtained.

Ethics approval Ethics approval was provided by the Royal Hospital for Sick Children Ethicas Committee.

Contributors RB, data analysis, writing and editing; GND (guarantor), initial concept, study design, planning, writing and editing; KM, planning, data collection and preliminary data analysis; EL, planning, data collection and preliminary data analysis; DLM (guarantor), study design, data collection, analysis, planning, writing and editing.

Provenance and peer review Not commissioned; externally peer reviewed.

\section{REFERENCES}

1. Teller DY, Mayer DL, Makous WL, et al. Do preferential looking techniques underestimate infant visual acuity. Vision Res 1982;22:1017-24.

2. Goren CC, Sarty M, Wu PY. Visual following and pattern discrimination of face-like stimuli by newborn infants. Pediatrics 1975;56:544-9.

3. Morton J, Johnson MH. CONSPEC and CONLERN: a two-process theory of infant face recognition. Psychol Rev 1991;98:164-81.

4. Teller DY. First glances: the vision of infants. the Friedenwald lecture. Invest Ophthalmol Vis Sci 1997;38:2183-203.

5. Atkinson J. The developing visual brain. Oxford Psychology Series No. 32. New York: Oxford University Press, c2000: 44-7. Chapter 4, Newborn vision.

6. Bullimore MA, Bailey IL, Wacker RT. Face recognition in age-related maculopathy. Invest Ophthalmol Vis Sci 1991;32:2020-9.

7. Tejeria $\mathbf{L}$, Harper RA, Artes $\mathrm{PH}$, et al. Face recognition in age related macular degeneration: perceived disability, measured disability, and performance with a bioptic device. Br J Ophthalmol 2002;86:1019-26.

8. Owsley C, Sloane ME. Contrast sensitivity, acuity, and the perception of 'real-world' targets. Br J Ophthalmol 1987;71:791-6.

9. Alexander MF, Maguire MG, Lietman TM, et al. Assessment of visual function in patients with age-related macular degeneration and low visual acuity. Arch Ophthalmol 1988;106:1543-7.

10. Lott LA, Haegerstrom-Portnoy G, Schneck ME, et al. Face recognition in the elderly. Optom Vis Sci 2005:82:874-81.

11. West SK, Rubin GS, Broman AT, et al. How does visual impairment affect performance on tasks of everyday life? The SEE Project. Salisbury Eye Evaluation. Arch Ophthalmol 2002;120:774-80.

12. Anderson JR. The development of self-recognition: a review. Dev Psychobiol 1984;17:35-49.

13. McDonald MA, Dobson V, Sebris SL, et al. The acuity card procedure: a rapid test of infant acuity. Invest Ophthalmol Vis Sci 1985;26:1158-62.

14. Hartmann EE, Ellis GS Jr, Morgan KS, et al. The acuity card procedure: Iongitudinal assessments. J Pediatr Ophthalmol Strabismus 1990;27:178-84.

15. Ellis GS Jr, Hartmann EE, Love A, et al. Teller acuity cards versus clinical judgment in the diagnosis of amblyopia with strabismus. Ophthalmology 1988;95:788-91

16. Lange C, Feltgen N, Junker B, et al. Resolving the clinical acuity categories 'hand motion' and 'counting fingers' using the Freiburg Visual Acuity Test (FrACT). Graefes Arch Clin Exp Ophthalmol 2009;247:137-42. 